\title{
ON THE POLAR QUOTIENTS OF AN ANALYTIC PLANE CURVE
}

\author{
Janusz Gwoździewicz and Arkadiusz Ploski
}

\begin{abstract}
We give explicit formulae in terms of characteristics and intersection multiplicities of branches for the polar quotients of a plane many-branched singularity.
\end{abstract}

\section{Introduction}

We use standard notations: $C\{X, Y\}$ is the ring of convergent power series, ord $f$ is the order of $f=f(X, Y) \in C\{X, Y\},(f, \phi)_{0}$ denotes the intersection multiplicity of $f$ with $\phi$. Recall that $(f, \phi)_{0} \geq(\operatorname{ord} f)(\operatorname{ord} \phi)$ with equality if and only if $f, \phi$ are transverse. We put ord $0=+\infty$ and use usual conventions on the symbol $+\infty$.

Let $f=f(X, Y) \in C\{X, Y\}$ be a reduced power series (i.e. without multiple factors) and let $t=t(X, Y) \in C\{X, Y\}$ be a regular parameter (i.e. a series of order 1) such that $t$ does not divide $f$. We consider the set of polar quotients of $f$ with respect to $t$ :

$$
Q(f, t)=\left\{\frac{(f, \phi)_{0}}{(t, \phi)_{0}}: \phi \text { is an irreducible factor of } \frac{\partial(t, f)}{\partial(X, Y)}\right\} .
$$

The polar quotients were studied by many authors $([\mathrm{T}],[\mathrm{M}],[\mathrm{E}],[\mathrm{Eph}]$, [Ca], [LMW1], [LMW2], [Ga], [D], [LP]). Recently, an explicit formula for the maximal polar quotient $q(f, t)=\sup Q(f, t)$ has been given ([P], Theorem 1.3). In this note we give similar formulae for all polar quotients. Like in $[\mathrm{P}]$ our main tool is the Kuo-Lu lemma ([KL], Lemma 3.3 and Section 2 of this paper). Instead of using Puiseux' trees (see $[\mathrm{KL}],[\mathrm{E}]$ ) we make our calculations by means of Puiseux' date (Section 3). The notion of symmetric power explained in [Wh], Appendix $\mathrm{V}$ turns out very useful.

Let $A$ be a nonempty set. Then $A_{\text {sym }}^{p}$ is the set of all $p$-tuples regarded as unordered. If $\alpha=\left\langle a_{1}, \ldots, a_{p}\right\rangle \in A_{\mathrm{sym}}^{p}$ then $|\alpha|=\left\{a_{1}, \ldots, a_{p}\right\}$ is the set corresponding to $\alpha$. We put $\operatorname{deg} \alpha=p$. If $\alpha=\left\langle a_{1}, \ldots, a_{p}\right\rangle$ and $\beta=\left\langle b_{1}, \ldots, b_{q}\right\rangle$

1991 Mathematics Subject Classification: 14H20, 32S10.

Key words and phrases: Plane curve singularity, polar quotients, Puiseux' date.

The second author was partially supported by the KBN grant P03A00115.

Received April 11, 2001. 
then $\alpha \oplus \beta=\left\langle a_{1}, \ldots, a_{p}, b_{1}, \ldots, b_{q}\right\rangle . \quad$ Clearly $|\alpha \oplus \beta|=|\alpha| \cup|\beta|$ and $\operatorname{deg}(\alpha \oplus \beta)=$ $\operatorname{deg} \alpha+\operatorname{deg} \beta$. For every positive integer $m$ we put $m \alpha=\alpha \oplus \cdots \oplus \alpha(m$ times). Instead of $m_{1}\left\langle a_{1}\right\rangle \oplus \cdots \oplus m_{p}\left\langle a_{p}\right\rangle=\langle\underbrace{a_{1}, \ldots, a_{1}}, \ldots, \underbrace{a_{p}, \ldots, a_{p}}\rangle \quad$ we write $\left\langle a_{1}: m_{1}, \ldots, a_{p}: m_{p}\right\rangle$.

\section{Main result}

The semigroup $\Gamma_{0}(f)$ of an irreducible power series $f \in C\{X, Y\}$ is the set of all intersection numbers $(f, \phi)_{0}$ where $\phi$ runs over all power series $\phi \in$ $C\{X, Y\}$ such that $f$ does not divide $\phi$. Let $t=t(X, Y)$ be a regular parameter. Put $p=(t, f)_{0}$. Let $\bar{b}_{0}, \ldots, \bar{b}_{h}$ be the $p$-minimal system of generators of $\Gamma_{0}(f)$ determined by conditions

(i) $\bar{b}_{0}=p$,

(ii) $\bar{b}_{k}=\min \left(\Gamma_{0}(f) \backslash\left(\boldsymbol{N} \bar{b}_{0}+\cdots+\boldsymbol{N} \bar{b}_{k-1}\right)\right)$ for $k=1, \ldots, h$,

(iii) $\Gamma_{0}(f)=\boldsymbol{N} \bar{b}_{0}+\cdots+\boldsymbol{N} \bar{b}_{h}$.

Let $B_{k}=\operatorname{GCD}\left(\bar{b}_{0}, \ldots, \bar{b}_{k}\right)$ be the greatest common divisor of $\bar{b}_{0}, \ldots, \bar{b}_{k}$.

Proposition 1.1 ([M], [Eph]). Let $f \in \boldsymbol{C}\{X, Y\}$ be an irreducible power series with ord $f>1$. Then

$$
Q(f, t)=\left\{\frac{B_{k-1} \bar{b}_{k}}{\bar{b}_{0}}: k=1, \ldots, h\right\} .
$$

The above formula was given by Merle ([M], Théorème 3.1) in transverse case. Then Ephraim ([Eph], Lemma 1.6) observed that Merle's result holds also for polar quotients with respect to a regular parameter $t$. In Section 4 we give a new proof of (1.1). Recall that the sequence $B_{k-1} \bar{b}_{k} / \bar{b}_{0}$ is strictly increasing.

If ord $f=1$ then $Q(f, t)=\emptyset$ if $f, t$ are transverse and $Q(f, t)=\{1\}$ if $f, t$ are not.

Remark 1.2. Using the inversion formulae for plane branches (see $[\mathrm{P}]$, proof of Proposition 1.1) one can calculate the polar quotients of a branch $f=0$ in terms of the minimal system of generators $\bar{\beta}_{0}, \ldots, \bar{\beta}_{q}$ of $\Gamma_{0}(f)$. Let $e_{k}=$ $\operatorname{GCD}\left(\bar{\beta}_{0}, \ldots, \bar{\beta}_{k}\right)$ for $k=0,1, \ldots, g$. Then

$$
Q(f, t)=\left\{\frac{e_{k-1} \bar{\beta}_{k}}{\bar{\beta}_{0}}: k=1, \ldots, g\right\} \cup O(f, t)
$$

where $O(f, t)=\emptyset$ if $(f, t)_{0}=\bar{\beta}_{0}$ or $(f, t)_{0}=\bar{\beta}_{1}$ and $O(f, t)=\{$ ord $f\}$ otherwise.

Let $\phi, \psi \in C\{X, Y\}$ be irreducible power series. The contact coefficient (in the sense of Hironaka) with respect to a regular parameter $t \in C\{X, Y\}$ is the rational number

$$
h(\phi, \psi ; t)=\frac{(\phi, \psi)_{0}}{(t, \psi)_{0}}
$$


In [LMW1] this notion is introduced in the case where $t$ and $\psi$ are transverse. Then $h(\phi, \psi ; t)=(\phi, \psi)_{0} /$ ord $\psi$ and we write $h(\phi, \psi)$ instead of $h(\phi, \psi ; t)$.

Let us consider a reduced power series $f=f_{1} \cdots f_{r}$ with $r>1$ irreducible factors. Fix $i \in\{1, \ldots, r\}$. For every real number $\tau>0$ we put $J_{i}(\tau)=$ $\left\{j \in\{1, \ldots, r\}: h\left(f_{i}, f_{j} ; t\right) \leq \tau\right\}$ and $J_{i}(\tau)^{c}=\{1, \ldots, r\} \backslash J_{i}(\tau)$.

By convention $h\left(f_{i}, f_{i} ; t\right)=+\infty$, hence $i \in J_{i}(\tau)^{c}$. We put

$$
q_{i}(\tau)=\frac{1}{\left(t, f_{i}\right)_{0}}\left\{\left(\sum_{j \in J_{i}(\tau)^{c}}\left(t, f_{j}\right)_{0}\right) \tau+\sum_{j \in J_{i}(\tau)}\left(f_{i}, f_{j}\right)_{0}\right\} \text { for } \tau>0 .
$$

To write the above formula more explicitly suppose $i=1$ and assume $h\left(f_{1}, f_{r} ; t\right) \leq h\left(f_{1}, f_{r-1} ; t\right) \leq \cdots \leq h\left(f_{1}, f_{2} ; t\right)<h\left(f_{1}, f_{1} ; t\right)=+\infty$. Then

$$
q_{1}(\tau)= \begin{cases}\frac{\left(t, f_{1} \cdots f_{j}\right)_{0}}{\left(t, f_{1}\right)_{0}} \tau+\frac{\left(f_{1}, f_{j+1} \cdots f_{r}\right)_{0}}{\left(t, f_{1}\right)_{0}} & \text { if } h\left(f_{1}, f_{j+1} ; t\right) \leq \tau<h\left(f_{1}, f_{j} ; t\right), j<r \\ \frac{(t, f)_{0}}{\left(t, f_{1}\right)_{0}} \tau & \text { if } \tau<h\left(f_{1}, f_{r} ; t\right)\end{cases}
$$

It is easy to see that the functions $q_{i}$ are piecewise linear, continuous and strictly increasing. Note that $q_{i}$ is determined by the intersection multiplicities of $i$-th branch $f_{i}=0$ with the remaining branches and with the smooth curve $t=0$. Let $H_{i}(f, t)=\left\{h\left(f_{i}, f_{j} ; t\right): j \neq i\right\}$ be the set of contact coefficients of the branch $f_{i}=0$ with branches $f_{j}=0(j \neq i)$. The main results of this paper is

THEOREM 1.3. Let $f=f_{1} \cdots f_{r}$ be a reduced power series with $r>1$ irreducible factors. Then

$$
Q(f, t)=\bigcup_{i=1}^{r} q_{i}\left(Q\left(f_{i}, t\right) \cup H_{i}(f, t)\right) .
$$

We give the proof of (1.3) in Section 4. We put $Q_{i}(f, t)=q_{i}\left(Q\left(f_{i}, t\right) \cup\right.$ $\left.H_{i}(f, t)\right)$ and call the elements of $Q_{i}(f, t)$ polar quotients associated with the branch $f_{i}=0$.

A polar quotient can be associated with more than one branch. From Theorem 1.3 it follows that the polar quotients of the curve $f=0$ with respect to a regular parameter $t$ depend only on the equisingularity type of the curve $t f=0$.

If $t$ and $f$ are transverse then the polar quotients of $f=0$ do not depend on the regular parameter $t$ and are determined by the equisingularity type of the curve $f=0$. Then we write $Q_{i}(f)$ instead of $Q_{i}(f, t)$ and $Q(f)$ instead of $Q(f, t)$.

Using (1.3) we obtain easily the formula for the maximal polar quotient $q(f, t)=\sup Q(f, t)$ given in $[\mathrm{P}]$. Obviously $q(f, t)=\max _{i=1}^{r}\left\{\max Q_{i}(f, t)\right\}$, hence it suffices to calculate the maximal polar quotients associated with branches. 
COROLlary 1.4 .

$$
\max Q_{i}(f, t)=\max \left\{q\left(f_{i}, t\right), \max _{j \neq i}\left\{h\left(f_{i}, f_{j} ; t\right)\right\}\right\}+\frac{1}{\left(t, f_{i}\right)_{0}} \sum_{j \neq i}\left(f_{i}, f_{j}\right)_{0} .
$$

Proof. Let $\tau_{i}=\max \left(Q\left(f_{i}, t\right) \cup H_{i}(f, t)\right)=\max \left\{q\left(f_{i}, t\right), \max _{j \neq i}\left\{h\left(f_{i}, f_{j} ; t\right)\right\}\right\}$. Then $J_{i}\left(\tau_{i}\right)=\{1, \ldots, r\} \backslash\{i\}$ and $q_{i}\left(\tau_{i}\right)=\tau_{i}+\left(1 /\left(t, f_{i}\right)_{0}\right) \sum_{j \neq i}\left(f_{i}, f_{j}\right)_{0}$. We get then $\max Q_{i}(f, t)=q_{i}\left(\tau_{i}\right)$ for $q_{i}$ is an increasing function.

Note that for every $i \in\{1, \ldots, r\}$ the rational numbers $q\left(f_{i}, t\right)+\left(1 /\left(t, f_{i}\right)_{0}\right)$. $\sum_{j \neq i}\left(f_{i}, f_{j}\right)_{0}$ (provided that $q\left(f_{i}, t\right) \neq-\infty$ ) and $\max _{j \neq i}\left\{h\left(f_{i}, f_{j} ; t\right)\right\}+\left(1 /\left(t, f_{i}\right)_{0}\right)$. $\sum_{j \neq i}\left(f_{i}, f_{j}\right)_{0}$ are polar quotients of $f=0$. then

COROllary 1.5. If $f=f_{1} \cdots f_{r}(r>1)$ with transverse factors $f_{i}, f_{j}$ for $i \neq j$

$$
Q(f)=\bigcup_{i=1}^{r}\left\{\tau_{i}+\text { ord } f-\text { ord } f_{i}: \tau_{i} \in Q\left(f_{i}\right)\right\} \cup\{\text { ord } f\} .
$$

Proof. Let us calculate $Q_{1}(f)$. It is easy to see that $J_{1}(\tau)=\{j \in\{2, \ldots, r\}$ : ord $\left.f_{1} \leq \tau\right\}$. Hence $J_{1}(\tau)=\emptyset$ if $\tau<$ ord $f_{1}$ and $J_{1}(\tau)=\{2, \ldots, r\}$ if $\tau \geq \operatorname{ord} f_{1}$. Obviously $H_{1}(f)=\left\{\right.$ ord $\left.f_{1}\right\}$. Therefore we get

$$
q_{1}(\tau)= \begin{cases}\tau+\operatorname{ord} f-\operatorname{ord} f_{1} & \text { if } \tau \geq \operatorname{ord} f_{1} \\ \frac{\operatorname{ord} f}{\operatorname{ord} f_{1}} \tau & \text { if } \tau<\operatorname{ord} f_{1}\end{cases}
$$

For every $\tau_{1} \in Q\left(f_{1}\right)$ one gets $\tau_{1} \geq$ ord $f_{1}$. Consequently $Q_{1}(f)=\left\{q_{1}\left(\tau_{1}\right): \tau_{1} \in\right.$ $\left.Q\left(f_{1}\right)\right\} \cup q_{1}\left(\right.$ ord $\left.f_{1}\right)=\left\{\tau_{1}+\right.$ ord $f-$ ord $\left.f_{1}: \tau_{1} \in Q\left(f_{1}\right)\right\} \cup\{$ ord $f\}$ and the corollary follows.

To give another application of the main result we calculate polar quotients of "diagonal curves" introduced by Delgado [D].

COROLlary 1.6. Let $f=f_{1} \cdots f_{r}$ be a reduced power series. Suppose that $\Gamma_{0}\left(f_{i}\right)$ and $\left(t, f_{i}\right)_{0}$ do not depend on $i \in\{1, \ldots, r\}, \Gamma_{0}\left(f_{i}\right)=\left\langle\bar{b}_{0}, \bar{b}_{1}, \ldots, \bar{b}_{h}\right\rangle$ and $\left(f_{i}, f_{j}\right)_{0}=B_{h-1} \bar{b}_{h}+c$ with $c>0$, for $i \neq j$. Then

$$
Q(f, t)=\left\{r \frac{B_{k-1} \bar{b}_{k}}{\bar{b}_{0}}: k=1, \ldots, h\right\} \cup\left\{r \frac{B_{h-1} \bar{b}_{h}+c}{\bar{b}_{0}}\right\} .
$$

Proof. By Proposition 1.1 we get $Q\left(f_{i}, t\right)=\left\{B_{k-1} \bar{b}_{k} / \bar{b}_{0}: k=1, \ldots, h\right\}$ on the other hand $H_{i}(f, t)=\left\{\left(B_{h-1} \bar{b}_{h}+c\right) / \bar{b}_{0}\right\}$. It is easy to check that $q_{i}(\tau)=r \tau$ if $\tau<\left(B_{h-1} \bar{b}_{h}+c\right) / \bar{b}_{0}$ and $q_{i}(\tau)=\tau+(r-1)\left(B_{h-1} \bar{b}_{h}+c\right) / \bar{b}_{0}$ if $\tau \geq\left(B_{h-1} \bar{b}_{h}+c\right) / \bar{b}_{0}$. Now we get (1.6) from Theorem 1.3. 


\section{Zeros of a derivative}

Let $\boldsymbol{C}\{X\}^{*}=\bigcup_{n \geq 1} \boldsymbol{C}\left\{X^{1 / n}\right\}$ be the ring of Puiseux series. If $f=f(X, Y) \in$ $\boldsymbol{C}\{X, Y\}$ is a power series $Y$-regular of order $p>0$ i.e. such that ord $f(0, Y)=p$ then the equation $f(X, Y)=0$ has in $C\{X\}^{*} p$ roots $y_{1}=y_{1}(X), \ldots, y_{p}=y_{p}(X)$ of positive order counted with multiplicities. We put Zer $f=\left\langle y_{1}, \ldots, y_{p}\right\rangle$. Let $S \subset \boldsymbol{C}\{X\}^{*}$ be a set of Puiseux series. If $y_{1}, \ldots, y_{q} \in S$ and $y_{q+1}, \ldots, y_{p} \notin S$ then we put Zer $f \cap S=\left\langle y_{1}, \ldots, y_{q}\right\rangle$. Let us recall

The Kuo-Lu Lemma ([KL], Lemma 3.3). Suppose that $f=f(X, Y)$ is $Y$-regular of order $p\rangle 1$. Let $\operatorname{Zer} f=\left\langle y_{1}, \ldots, y_{p}\right\rangle$ and $\operatorname{Zer}(\partial f / \partial Y)=$ $\left\langle z_{1}, \ldots, z_{p-1}\right\rangle$. Then for every $i \in\{1, \ldots, p\}$ :

$$
\begin{aligned}
& \left\langle\operatorname{ord}\left(z_{1}-y_{i}\right), \ldots, \operatorname{ord}\left(z_{p-1}-y_{i}\right)\right\rangle \\
& \quad=\left\langle\operatorname{ord}\left(y_{1}-y_{i}\right), \ldots, \operatorname{ord}\left(\widehat{y_{i}}-y_{i}\right), \ldots, \operatorname{ord}\left(y_{p}-y_{i}\right)\right\rangle .
\end{aligned}
$$

It is convenient to prove the lemma for formal power series with coefficients in $\boldsymbol{C}\{X\}^{*}$. Replacing $f(X, Y)$ by $f\left(X, Y+y_{i}\right)$ we may assume $y_{i}=0$. Then the series $f(X, Y) / Y$ and $\partial f / \partial Y$ have the same Newton diagram (see [Wa], Chapter IV) and the lemma follows from the fact that the Newton diagram determines the orders of roots and the number of roots of given order. For more details see [GP1].

For every $u \in C\{X\}^{*}$ and $r>0$ we put

$$
\begin{aligned}
& S(u, r)=\left\{v \in C\{X\}^{*}: \operatorname{ord}(u-v)=r\right\}, \\
& B(u, r)=\left\{v \in C\{X\}^{*}: \operatorname{ord}(u-v)>r\right\}, \\
& \bar{B}(u, r)=\left\{v \in C\{X\}^{*}: \operatorname{ord}(u-v) \geq r\right\} .
\end{aligned}
$$

According to the given definitions $\operatorname{deg}(\operatorname{Zer} f \cap S)$ is equal to the number of roots counted with multiplicities of the equation $f(X, Y)=0$ lying in the set $S$ of Puiseux series. If $\mid$ Zer $f \mid \cap S=\emptyset$ then we put $\operatorname{deg}(\operatorname{Zer} f \cap S)=0$.

Lemma 2.1. Suppose that $f=f(X, Y)$ is $Y$-regular of order $p>1$. Then for every $y \in \operatorname{Zer} f$ and for every $r>0$

(i) $\operatorname{deg}(\operatorname{Zer} f \cap S(y, r))=\operatorname{deg}(\operatorname{Zer}(\partial f / \partial Y) \cap S(y, r))$,

(ii) $\operatorname{deg}(\operatorname{Zer} f \cap B(y, r))=\operatorname{deg}(\operatorname{Zer}(\partial f / \partial Y) \cap B(y, r))+1$,

(iii) $\operatorname{deg}(\operatorname{Zer} f \cap \bar{B}(y, r))=\operatorname{deg}(\operatorname{Zer}(\partial f / \partial Y) \cap \bar{B}(y, r))+1$.

Proof. The part (i) is a reformulation of the Kuo-Lu lemma, (ii) and (iii) follow from (i).

Proposition 2.2. Under assumptions of Lemma 2.1:

(i) for every $z \in|\operatorname{Zer}(\partial f / \partial Y)|$ there exist $y_{1}, y_{2} \in \operatorname{Zer} f$, $y_{1} \neq y_{2}$ such that $\operatorname{ord}\left(z-y_{1}\right)=\operatorname{ord}\left(y_{1}-y_{2}\right) \geq \operatorname{ord}(z-y)$ for all $y \in \mid$ Zer $f \mid$, 
(ii) for every $y_{1}, y_{2} \in \operatorname{Zer} f, \quad y_{1} \neq y_{2}$ there exists $z \in|\operatorname{Zer}(\partial f / \partial Y)|$ such that $\operatorname{ord}\left(z-y_{1}\right)=\operatorname{ord}\left(y_{1}-y_{2}\right) \geq \operatorname{ord}(z-y)$ for all $y \in \mid$ Zer $f \mid$.

Proof. (i) Fix $z \in|\operatorname{Zer}(\partial f / \partial Y)|$. Let $y_{1} \in|\operatorname{Zer} f|$ be such that $\operatorname{ord}\left(z-y_{1}\right)=$ $\max \{\operatorname{ord}(z-y): y \in \mid$ Zer $f \mid\}$. By the Kuo-Lu lemma there exists $y_{2} \in \mid$ Zer $f \mid$ such that $\operatorname{ord}\left(z-y_{1}\right)=\operatorname{ord}\left(y_{1}-y_{2}\right)$ and we are done.

(ii) Suppose $y_{1} \neq y_{2}$ are given roots of the equation $f(X, Y)=0$.

Let $\bar{B}=\bar{B}\left(y_{1}, \operatorname{ord}\left(y_{1}-y_{2}\right)\right)$. Let $B_{1}, \ldots, B_{s}$ be a sequence of pairwise disjoint balls such that $\bigcup_{i=1}^{s} B_{i}=\bigcup\left\{B\left(y, \operatorname{ord}\left(y_{1}-y_{2}\right)\right): y \in \mid\right.$ Zer $\left.f \mid \cap \bar{B}\right\}$.

Note that

1) $s \geq 2$ for $B\left(y_{1}, \operatorname{ord}\left(y_{1}-y_{2}\right)\right) \cap B\left(y_{2}, \operatorname{ord}\left(y_{1}-y_{2}\right)\right)=\emptyset$,

2) $B_{i} \subset \bar{B}(i=1, \ldots, s)$ because the center of $B_{i}$ belongs to $\bar{B}$ and $B_{i}, \bar{B}$ have the same radius.

3) $\bigcup_{i=1}^{s}\left(B_{i} \cap \mid\right.$ Zer $\left.f \mid\right)=\bar{B} \cap \mid$ Zer $f \mid$ for $B_{i}$ cover $\bar{B} \cap \mid$ Zer $f \mid$ and are pairwise disjoint.

Using Lemma 2.1 we calculate $\operatorname{deg}\left(\left(\bar{B} \backslash \bigcup_{i=1}^{s} B_{i}\right) \cap \operatorname{Zer}(\partial f / \partial Y)\right)=$ $\operatorname{deg}(\bar{B} \cap \operatorname{Zer}(\partial f / \partial Y))-\sum_{i=1}^{s} \operatorname{deg}\left(B_{i} \cap \operatorname{Zer}(\partial f / \partial Y)\right)=(\operatorname{deg}(\bar{B} \cap \operatorname{Zer} f)-1)$ $-\sum_{i=1}^{s}\left(\operatorname{deg}\left(B_{i} \cap \operatorname{Zer} f\right)-1\right)=\operatorname{deg}(\bar{B} \cap$ Zer $f)-\sum_{i=1}^{s} \operatorname{deg}\left(B_{i} \cap\right.$ Zer $\left.f\right)+$ $(s-1)=s-1>0$.

Therefore the set $\left(\bar{B} \backslash \bigcup_{i=1}^{s} B_{i}\right) \cap|\operatorname{Zer}(\partial f / \partial Y)|$ is nonempty. Let $z \in$ $\left(\bar{B} \backslash \bigcup_{i=1}^{s} B_{i}\right) \cap|\operatorname{Zer}(\partial f / \partial Y)|$. Then $\operatorname{ord}\left(z-y_{1}\right) \geq \operatorname{ord}\left(y_{1}-y_{2}\right) \geq \operatorname{ord}(z-y)$ for all $y \in \mid$ Zer $f \mid \cap \bar{B}$. In particular $\operatorname{ord}\left(z-y_{1}\right)=\operatorname{ord}\left(y_{1}-y_{2}\right)$. If $y \in|\operatorname{Zer} f| \backslash \bar{B}$ then $\operatorname{ord}\left(y-y_{1}\right)<\operatorname{ord}\left(y_{1}-y_{2}\right)=\operatorname{ord}\left(z-y_{1}\right)$. Hence we get $\operatorname{ord}\left(z-y_{1}\right) \geq$ $\operatorname{ord}(z-y)$ for all $y \in \mid$ Zer $f \mid$. Thus (ii) follows.

For given $y_{1}, y_{2} \in|\operatorname{Zer} f|, y_{1} \neq y_{2}$ we put

$$
\begin{gathered}
L_{y_{1}, y_{2}}=|\operatorname{Zer} f| \cap \bar{B}\left(y_{1}, \operatorname{ord}\left(y_{1}-y_{2}\right)\right), \\
L_{y_{1}, y_{2}}^{c}=\sum_{y \in L_{y_{1}, y_{2}}^{c}, y_{2}} \operatorname{ord}\left(y_{1}-y\right)+\left(\sharp L_{y_{1}, y_{2}}\right) \operatorname{ord}\left(y_{1}-y_{2}\right) .
\end{gathered}
$$

THEOREM 2.3. Let $f=f(X, Y)$ be a reduced $Y$-regular power series with ord $f(0, Y)>1$. Then $\left\{l_{y_{1}, y_{2}}: y_{1}, y_{2} \in|\operatorname{Zer} f|, y_{1} \neq y_{2}\right\}=\{$ ord $f(X, z(X)): z \in$ $|\operatorname{Zer}(\partial f / \partial Y)|\}$.

Proof. For every $z=z(X) \in C\{X\}^{*}$ without constant term one has

$$
\text { ord } f(X, z(X))=\sum_{y \in|\operatorname{Zer} f|} \operatorname{ord}(z-y)=\sum_{y \in L_{y_{1}, y_{2}}} \operatorname{ord}(z-y)+\sum_{y \in L_{y_{1}, y_{2}}} \operatorname{ord}(z-y) .
$$

Let $y_{1}, y_{2} \in \operatorname{Zer} f$ and $z \in|\operatorname{Zer}(\partial f / \partial Y)|$ be such that $\operatorname{ord}\left(z-y_{1}\right)=\operatorname{ord}\left(z-y_{2}\right) \geq$ $\operatorname{ord}(z-y)$ for all $y \in|\operatorname{Zer} f|$. We will check that

$$
\operatorname{ord}(z-y)= \begin{cases}\operatorname{ord}\left(y-y_{1}\right) & \text { if } y \in L_{y_{1}, y_{2}}^{c} \\ \operatorname{ord}\left(y_{2}-y_{1}\right) & \text { if } y \in L_{y_{1}, y_{2}}\end{cases}
$$


Indeed, if $y \in L_{y_{1}, y_{2}}^{c}$ then $\operatorname{ord}(z-y)=\operatorname{ord}\left(y-y_{1}\right)$ for $\operatorname{ord}\left(y-y_{1}\right)<\operatorname{ord}\left(y_{2}-y_{1}\right)$ $=\operatorname{ord}\left(z-y_{1}\right)$.

If $y \in L_{y_{1}, y_{2}}$ then

$$
\begin{aligned}
\operatorname{ord}(z-y) \geq \min \left\{\operatorname{ord}\left(z-y_{1}\right), \operatorname{ord}\left(y_{1}-y\right)\right\} & =\min \left\{\operatorname{ord}\left(y_{2}-y_{1}\right), \operatorname{ord}\left(y_{1}-y\right)\right\} \\
& =\operatorname{ord}\left(y_{2}-y_{1}\right) .
\end{aligned}
$$

On the other hand $\operatorname{ord}(z-y) \leq \operatorname{ord}\left(z-y_{1}\right)$ for all $y \in \mid$ Zer $f \mid$ and $\operatorname{ord}(z-y) \leq$ $\operatorname{ord}\left(y_{2}-y_{1}\right)$. Hence $\operatorname{ord}(z-y)=\operatorname{ord}\left(y_{2}-y_{1}\right)$ for $y \in L_{y_{1}, y_{2}}$. Now, Theorem 2.3 follows from (1), (2) and Proposition 2.2.

\section{Puiseux' date}

We use the notations and definitions from $[\mathrm{P}]$, Section 3. Let $f=$ $f(X, Y) \in \boldsymbol{C}\{X, Y\}$ be an irreducible power series $Y$-regular of order $p>0$. Let $\left(b_{0}, \ldots, b_{h}\right)$ be the characteristic of $f$. We put $B_{k}=\operatorname{GCD}\left(b_{0}, \ldots, b_{k}\right)$ for $k=0,1, \ldots, h \quad$ and $\quad \bar{b}_{k}=b_{k}+\left(1 / B_{k-1}\right) \sum_{i=1}^{k-1}\left(B_{i-1}-B_{i}\right) b_{i}$ for $k=0,1, \ldots, h$. Then the sequence $\bar{b}_{0}=p, \bar{b}_{1}, \ldots, \bar{b}_{h}$ is the $p$-minimal system of generators of the semigroup $\Gamma_{0}(f)$ (see $[\mathrm{P}]$, Proposition 3.2). We put $P(f)=\left\langle b_{1} / b_{0}\right.$ : $\left.B_{0}-B_{1}, \ldots, b_{h} / b_{0}: B_{h-1}-B_{h},+\infty: 1\right\rangle$ and call $P(f)$ Puiseux' date of the branch $f=0$. Clearly $|P(f)|=\left\{b_{1} / b_{0}, \ldots, b_{h} / b_{0},+\infty\right\}$. If $p=1$ then $P(f)=\langle+\infty\rangle$.

Proposition 3.1. Let Zer $f=\left\langle y_{1}, \ldots, y_{p}\right\rangle$. Then for every $i \in\{1, \ldots, p\}$ :

$$
\left\langle\operatorname{ord}\left(y_{1}-y_{i}\right), \ldots, \operatorname{ord}\left(y_{p}-y_{i}\right)\right\rangle=P(f) \text {. }
$$

Proof. It is a reformulation of Proposition 3.1 from $[\mathrm{P}]$.

The following notation will be useful. Let $\alpha \in \boldsymbol{R}_{\text {sym }}^{p}$ and let $K>0$ be a real number. Write $\alpha=\left\langle a_{1}, \ldots, a_{q}, a_{q+1}, \ldots, a_{p}\right\rangle$ where $a_{1}, \ldots, a_{q}<K$ and $K \leq$ $a_{q+1}, \ldots, a_{p}$. Then we put $\alpha_{K}=\left\langle a_{1}, \ldots, a_{q}, K, \ldots, K\right\rangle \in \boldsymbol{R}_{\text {sym }}^{p}$ with $K$ repeated $p-q$ times. We let $\alpha_{(+\infty)}=\alpha$. Clearly $(\alpha \oplus \beta)_{K}=\alpha_{K} \oplus \beta_{K}$ and $\left(\alpha_{K}\right)_{K^{\prime}}=$ $\alpha_{\min \left(K, K^{\prime}\right)}$.

Let $g=g(X, Y) \in C\{X, Y\}$ be an irreducible $Y$-regular power series. Let Zer $g=\left\langle z_{1}(X), \ldots, z_{q}(X)\right\rangle$. Then the order of contact of $f$ and $g$ is defined to be

$$
\operatorname{cont}(f, g)=\max \left\{\operatorname{ord}\left(y_{i}(X)-z_{j}(X)\right): 1 \leq i \leq p, 1 \leq j \leq q\right\} .
$$

Note that for every $K>0$

$$
P(f)_{K}=\left\langle\frac{b_{1}}{b_{0}}: B_{0}-B_{1}, \ldots, \frac{b_{k-1}}{b_{0}}: B_{k-2}-B_{k-1}, K: B_{k-1}\right\rangle
$$

where $k>0$ is the smallest integer such that $K \leq b_{k} / b_{0}$. We put $b_{h+1} / b_{0}=+\infty$.

Proposition 3.2. Let $z=z(X) \in \mid$ Zer $g \mid$. Then

$$
\left\langle\operatorname{ord}\left(z-y_{1}\right), \ldots, \operatorname{ord}\left(z-y_{p}\right)\right\rangle=P(f)_{\operatorname{cont}(f, g)} \text {. }
$$


Proof. It is easy to check that $\max _{i=1}^{p}\left\{\operatorname{ord}\left(z-y_{i}\right)\right\}=\operatorname{cont}(f, g)$. We may assume that $\operatorname{cont}(f, g)=\operatorname{ord}\left(z-y_{p}\right)$. Then

$$
\left\langle\operatorname{ord}\left(z-y_{1}\right), \ldots, \operatorname{ord}\left(z-y_{p}\right)\right\rangle=\left\langle\operatorname{ord}\left(y_{1}-y_{p}\right), \ldots, \operatorname{ord}\left(y_{p}-y_{p}\right)\right\rangle_{\operatorname{ord}\left(z-y_{p}\right)}
$$

and (3.2) follows from (3.1).

For every $\alpha=\left\langle a_{1}, \ldots, a_{p}\right\rangle \in \boldsymbol{R}_{\text {sym }}^{p}$ we put $\sum \alpha=\sum_{i=1}^{p} a_{i} . \quad$ Clearly $\sum(\alpha \oplus \beta)=$ $\sum \alpha+\sum \beta$. Note that $K \mapsto \sum \alpha_{K}$ is strictly increasing for $K>0$.

Proposition 3.3. Let $f=f(X, Y) \in \boldsymbol{C}\{X, Y\}$ and $g=g(X, Y) \in \boldsymbol{C}\{X, Y\}$ be irreducible $Y$-regular power series. Suppose $f$ is of characteristic $\left(b_{0}, \ldots, b_{h}\right)$. Then

(i) $\sum P(f)_{b_{k} / b_{0}}=B_{k-1} \bar{b}_{k} / \bar{b}_{0}$

(ii) $\sum P(f)_{\operatorname{cont}(f, g)}=(f, g)_{0} /(X, g)_{0}$,

(iii) $(X, f)_{0} P(g)_{\operatorname{cont}(f, g)}=(X, g)_{0} P(f)_{\operatorname{cont}(f, g)}$,

(iv) if $K \leq \operatorname{cont}(f, g)$ then $\sum P(g)_{K}=\left((X, g)_{0} /(X, f)_{0}\right) \sum P(f)_{K}$.

Proof. Property (i) follows from the definitions. To check (ii) recall that $(f, g)_{0} /(X, g)_{0}=$ ord $f(X, z(X))$ where $z=z(X) \in \mid$ Zer $g \mid$.

Hence $(f, g)_{0} /(X, g)_{0}=\sum_{i=1}^{p} \operatorname{ord}\left(z(X)-y_{i}(X)\right)=\sum P(f)_{\operatorname{cont}(f, g)}$ by Proposition 3.2.

Let $k>0$ be the smallest integer such that $\operatorname{cont}(f, g) \leq b_{k} / b_{0}$ and let us consider the characteristic $\left(b_{0}^{\prime}, \ldots, b_{h^{\prime}}^{\prime}\right)$ of $g$. Then $k \leq h^{\prime}, \operatorname{cont}(f, g) \leq b_{k}^{\prime} / b_{0}^{\prime}$ and $b_{i}^{\prime} / b_{0}^{\prime}=b_{i} / b_{0}$ for $i<k$. Consequently $b_{0}^{\prime} B_{i}=b_{0} B_{i}^{\prime}$ for $i \leq k$ and (iii) follows. Property (iv) is an easy consequence of (iii).

Remark 3.4. Properties (i), (ii) and (iii) listed in Proposition 3.3 are equivalent to the classical formula for the intersection multiplicity of two branches (see $[\mathrm{P}])$.

Consider a reduced power series $f=f_{1} \cdots f_{r}$ with $r>1$ factors. For every $i \in\{1, \ldots, r\}$ we put $K_{i j}=\operatorname{cont}\left(f_{i}, f_{j}\right)$ if $i \neq j$ and $K_{i i}=+\infty$. We define Puiseux' date $P_{i}(f)$ of $f$ with respect to the irreducible factor $f_{i}$ by putting

$$
P_{i}(f)=P\left(f_{1}\right)_{K_{i 1}} \oplus \cdots \oplus P\left(f_{r}\right)_{K_{i r}} \text {. }
$$

Proposition 3.5 justifies our definition of Puiseux' date.

Proposition 3.5. With the notation introduced above

(i) $\left|P_{i}(f)\right|=\left|P\left(f_{i}\right)\right| \cup\left\{K_{i 1}, \ldots, K_{i r}\right\}$,

(ii) Let $y_{i} \in \mid$ Zer $f_{j} \mid$ for some $j \in\{1, \ldots, r\}$. Then

$$
\left\langle\operatorname{ord}\left(y_{i}-y_{1}\right), \ldots, \operatorname{ord}\left(y_{i}-y_{i}\right), \ldots, \operatorname{ord}\left(y_{i}-y_{p}\right)\right\rangle=P_{j}(f) \text {. }
$$

Proof. We have $\left|P_{i}(f)\right|=\bigcup_{j=1}^{r}\left|P\left(f_{j}\right)_{K_{i j}}\right|=\bigcup_{j=1}^{r}\left|P\left(f_{i}\right)_{K_{i j}}\right|$ by Proposition 3.3(iii). Let $b_{0}, \ldots, b_{h}$ be the characteristic of the branch $f_{i}$. Then $\left|P\left(f_{i}\right)_{K_{i i}}\right|=$ 
$\left|P\left(f_{i}\right)\right|=\left\{b_{1} / b_{0}, \ldots, b_{h} / b_{0},+\infty\right\}$ and $\left|P\left(f_{i}\right)_{K_{i j}}\right|=\left\{b_{1} / b_{0}, \ldots, b_{k(j)-1} / b_{0}, K_{i j}\right\}$ where $k(j)$ is the smallest integer $k>0$ such that $K_{i j} \leq b_{k} / b_{0}$. Summing up we get $\left|P\left(f_{i}\right)\right|=\left\{b_{1} / b_{0}, \ldots, b_{h} / b_{0}, K_{i 1}, \ldots, K_{i r}\right\}$ which proves (i). Part (ii) follows from (3.1), (3.2) and from the definition of $P_{j}(f)$.

\section{Proof}

A local isomorphism $\Phi$ is a pair of power series without constant term such that $\operatorname{Jac} \Phi(0,0) \neq 0$. It is easy to check the following

Lemma 4.1. Let $\Phi$ be a local isomorphism. A rational number $q$ is a polar quotient of $f$ with respect to a regular parameter $t$ if and only if $q$ is a polar quotient of $f \circ \Phi$ with respect to $t \circ \Phi$.

Therefore to prove (1.1) and (1.3) it suffices to consider the case $t=X$.

Proposition 4.2. Let $f=f_{1} \cdots f_{r}$ be a reduced power series with irreducible factors $f_{i}$. Suppose that $f$ is $Y$-regular of order $p>0$ and let $p_{i}: \boldsymbol{R}_{+} \rightarrow \boldsymbol{R}$ be given by the formula

$$
\begin{array}{cl}
p_{i}(K)=\sum P_{i}(f)_{K} & \text { for } K>0 . \\
\text { Let } p_{i}\left(\left|P_{i}(f)\right|\right)=\left\{p_{i}(K): K \in\left|P_{i}(f)\right| \cap \boldsymbol{R}\right\} . & \text { Then } Q(f, X)=\bigcup_{i=1}^{r} p_{i}\left(\left|P_{i}(f)\right|\right) .
\end{array}
$$

Proof. Let $\operatorname{Zer} f=\left\langle y_{1}, \ldots, y_{p}\right\rangle$ and $\operatorname{Zer}(\partial f / \partial Y)=\left\langle z_{1}, \ldots, z_{p-1}\right\rangle$. It is easy to see that $Q(f, X)=\left\{\operatorname{ord} f\left(X, z_{1}(X)\right), \ldots\right.$ ord $\left.f\left(X, z_{p-1}(X)\right)\right\}$. Indeed, if $\phi$ is an irreducible factor of $\partial f / \partial Y$ and $z_{i}=z_{i}(X) \in \operatorname{Zer} \phi$ then $(f, \phi)_{0} /(X, \phi)_{0}=$ ord $f\left(X, z_{i}(X)\right)$.

Let $l_{y_{i}, y_{j}}(i \neq j)$ be the quantities introduced in Section 3 of this paper. It is easy to see that

$$
l_{y_{i}, y_{j}}=\sum\left\langle\operatorname{ord}\left(y_{i}-y_{1}\right), \ldots, \operatorname{ord}\left(y_{i}-y_{p}\right)\right\rangle_{\operatorname{ord}\left(y_{i}-y_{j}\right)}
$$

for $i \neq j$.

Now suppose that $y_{i} \in\left|\operatorname{Zer} f_{1}\right|$ and let $K_{1}=\operatorname{ord}\left(y_{i}-y_{j}\right)$ for a $j \neq i$. By Proposition 3.5 we get

$$
l_{y_{i}, y_{j}}=\sum P_{1}(f)_{K_{1}}=p_{1}\left(K_{1}\right)
$$

and consequently $\left\{l_{y_{i}, y_{j}}: j \neq i\right\}=\left\{p_{1}\left(K_{1}\right): K_{1} \in\left|P_{1}(f)\right|\right\}=p_{1}\left(\left|P_{1}(f)\right|\right)$.

Similarly $\quad\left\{l_{y_{i}, y_{j}}: j \neq i\right\}=p_{k}\left(\left|P_{k}(f)\right|\right) \quad$ if $\quad y_{i} \in\left|\operatorname{Zer} f_{k}\right|$ for $k=2, \ldots, r$. Therefore by Theorem 2.3 we get $Q(f, X)=\left\{l_{y_{i}, y_{j}}: j \neq i\right\}=p_{1}\left(\left|P_{1}(f)\right|\right) \cup \cdots \cup$ $p_{r}\left(\left|P_{r}(f)\right|\right)$.

Now we can give

Proof of Proposition 1.1. We assume $t=X$. Let $f$ be an irreducible power series $Y$-regular of order $p>1$. Let $\left(b_{0}, b_{1}, \ldots, b_{h}\right)$ be the characteristic of $f$. 
According to Proposition 4.2 we have $Q(f, X)=p(|P(f)|)$ where $p: \boldsymbol{R}_{+} \rightarrow \boldsymbol{R}$ is given by the formula $p(K)=\sum P(f)_{K}$ for $K>0$. Recall that $|P(f)|=$ $\left\{b_{1} / b_{0}, \ldots, b_{h} / b_{0},+\infty\right\}$ and $p\left(b_{k} / b_{0}\right)=\sum P(f)_{b_{k} / b_{0}}=B_{k-1} \bar{b}_{k} / \bar{b}_{0}$ by Proposition 3.3(i). Therefore $Q(f, X)=\left\{B_{k-1} \bar{b}_{k} / \bar{b}_{0}: k=1, \ldots, h\right\}$.

Proof of Theorem 1.3. We assume $t=X$. We need to calculate $p_{i}\left(\left|P_{i}(f)\right|\right)$ for $i=1, \ldots, r$. Without restriction of generality we assume $i=1$. Let $K>0$. By definition of Puiseux' date we get

$$
P_{1}(f)_{K}=P\left(f_{1}\right)_{\min \left(K, K_{11}\right)} \oplus \cdots \oplus P\left(f_{r}\right)_{\min \left(K, K_{1 r}\right)}
$$

Therefore

$$
\begin{aligned}
p_{1}(K) & =\sum P_{1}(f)_{K}=\sum_{j=1}^{r}\left(\sum P\left(f_{j}\right)_{\min \left(K, K_{1 j}\right)}\right) \\
& =\sum_{j=1}^{r} \frac{\left(X, f_{j}\right)_{0}}{\left(X, f_{1}\right)_{0}}\left(\sum P\left(f_{1}\right)_{\min \left(K, K_{1 j}\right)}\right)
\end{aligned}
$$

by Proposition 3.3(iv).

Recall that $K_{1 j} \leq K$ if and only if $\sum P\left(f_{1}\right)_{K_{1 j}} \leq \sum P\left(f_{1}\right)_{K}$. On the other hand by Proposition 3.3(ii) we get

$$
\sum P\left(f_{1}\right)_{K_{1 j}}=\frac{\left(f_{1}, f_{j}\right)_{0}}{\left(X, f_{j}\right)_{0}}
$$

Let $p_{11}(K)=\sum P\left(f_{1}\right)_{K}$. Thus $K_{1 j} \leq K$ if and only if $\left(f_{1}, f_{j}\right)_{0} /\left(X, f_{j}\right)_{0} \leq$ $p_{11}(K)$. By definition of the set $J_{1}(\tau)$ we see that $K_{1 j} \leq K$ if and only if $j \in J_{1}\left(p_{11}(K)\right)$. Then, we can rewrite the formula for $p_{1}(K)$ as follows

$$
\begin{aligned}
p_{1}(K) & =\sum_{j=1}^{r} \frac{\left(X, f_{j}\right)_{0}}{\left(X, f_{1}\right)_{0}}\left(\sum P\left(f_{1}\right)_{\min \left(K, K_{1 j}\right)}\right) \\
& =\sum_{j \in J_{1}^{c}\left(p_{11}(K)\right)} \frac{\left(X, f_{j}\right)_{0}}{\left(X, f_{1}\right)_{0}}\left(\sum P\left(f_{1}\right)_{\min \left(K, K_{1 j}\right)}\right)+\sum_{j \in J_{1}\left(p_{11}(K)\right)}\left(\sum P\left(f_{1}\right)_{\min \left(K, K_{1 j}\right)}\right) \\
& =\sum_{j \in J_{1}^{c}\left(p_{11}(K)\right)} \frac{\left(X, f_{j}\right)_{0}}{\left(X, f_{1}\right)_{0}} p_{11}(K)+\sum_{j \in J_{1}\left(p_{11}(K)\right)} \frac{\left(X, f_{j}\right)_{0}}{\left(X, f_{1}\right)_{0}} \frac{\left(f_{1}, f_{j}\right)_{0}}{\left(X, f_{j}\right)_{0}} \\
& \left.=\sum_{j \in J_{1}^{c}\left(p_{11}(K)\right)} \frac{\left(X, f_{j}\right)_{0}}{\left(X, f_{1}\right)_{0}}\right) p_{11}(K)+\sum_{j \in J_{1}\left(p_{11}(K)\right)} \frac{\left(f_{1}, f_{j}\right)_{0}}{\left(X, f_{1}\right)_{0}}=q_{1}\left(p_{11}(K)\right) .
\end{aligned}
$$

Recall that $\left|P_{1}(f)\right|=\left|P\left(f_{1}\right)\right| \cup\left\{K_{12}, \ldots, K_{1 r}\right\}$. Thus $\quad p_{11}\left(\left|P_{1}(f)\right|\right)=$ $p_{11}\left(\left|P_{1}(f)\right|\right) \cup\left\{p_{11}\left(K_{12}\right), \ldots, p_{11}\left(K_{1 r}\right)\right\}=Q\left(f_{1}, X\right) \cup H_{1}(f, X) \quad$ by Proposition 3.3(i), (ii) and $p_{1}\left(\left|P_{1}(f)\right|\right)=q_{1}\left(p_{11}\left(\left|P_{1}(f)\right|\right)\right)=q_{1}\left(Q\left(f_{1}, X\right) \cup H_{1}(f, X)\right)$. Analo- 
gously we get $p_{i}\left(\left|P_{i}(f)\right|\right)=q_{i}\left(Q\left(f_{i}, X\right) \cup H_{i}(f, X)\right)$ for $i=2, \ldots, r$ and the theorem follows from Proposition 4.2.

\section{REFERENCES}

[CA] E. Casas-Alvero, Singularities of Plane Curves, London Math. Soc. Lecture Note Ser. 276, Cambridge Univ. Press, Cambridge, 2000.

[D] F. Delgado de la Mata, A factorization theorem for the polar of a curve with two branches, Compositio Math., 92 (1994), 327-375.

[E] H. EgGers, Polarinvarianten und die Topologie von Kurvensingularitäten, Bonner Math. Schriften 147, Universität Bonn, Bonn, 1982.

[Eph] R. Ephraim, Special polars and curves with one place at infinity, Singularities (P. Orlik ed.), Proc. Sympos. Pure Math. 40 Part 1, AMS, Providence, 1983, 353-359.

[Ga] E. García Barroso, Sur les courbes polaires d'une courbe plane réduite, Proc. London Math. Soc. (3), 81 (2000), 1-28.

[GP1] J. GwoźDziewicz And A. Ploski, On the Merle formula for polar invariants, Bull. Soc. Sci. Lett. Łódź, 41 (1991), 61-67.

[GP2] J. GwoźDzIEWICZ AND A. Pleoski, On the approximate roots of polynomials, Ann. Polon. Math., 60 (1995), 199-210.

$[\mathrm{KL}] \quad$ T. C. Kuo AND Y. C. LU, On analytic function germs of two complex variables, Topology, 16 (1977), 299-310.

[LMW1] L. D. Trang, F. Michel et C. Weber, Sur le comportement des polaires associées aux germes de courbes planes, Compositio Math., 72 (1989), 87-113.

[LMW2] L. D. Trang, F. Michel et C. Weber, Courbes polaires et topologie des courbes planes, Ann. Sci. École Norm. Sup. (4), 24 (1991), 141-169.

[LP] A. Lenarcik and A. Ploski, Polar invariants of plane curves and the Newton polygon, Kodai Math. J., 23 (2000), 309-319.

[M] M. MerLe, Invariants polaires des courbes planes, Invent. Math., 41 (1977), 103-111.

[P] A. Ploski, On the maximal polar quotient of an analytic plane curve, Kodai Math. J., 24 (2001), 120-133.

[T] B. TeIssier, Variétés polaires. I. Invariants polaires des singularités d'hypersurfaces, Invent. Math., 40 (1977), 267-292.

[Wa] R. Walker, Algebraic Curves, Princeton Mathematical Series 13, Princeton Univ. Press, Princeton, 1950.

[Wh] H. Whitney, Complex Analytic Varieties, Addison-Wesley, Reading, 1972.

Department of Mathematics

TECHNICAL UNIVERSITY

AL. 1000 LPP 7

25-314 KIELCE

POLAND

e-mail: matjg@tu.kielce.pl

Department of Mathematics

TeChNICAL UNIVERSITY

AL. 1000 LPP 7

25-314 KIELCE

POLAND

e-mail: matap@tu.kielce.pl 